the same practice from independent observation, and from their own private experience. Dr. Todd acted upon this plan, as he would have acted upon any other, because he had obtained sufficient evidence to convince himself that it was right. Why too, I would ask, should a writer who has been called upon to review an important work, go far out of his way to annoy his readers by offering what almost amounts to an indirect insult to two distinguished physicians who are not in any way connected with the question at issue?

But, gentlemen, we must not pursue this part of the question further. We may hope that the time is not far distant when medical literature will no longer be degraded with this sort of writing; and I trust you will all uphold, on every opportunity, the propriety of discussing difficult questions freely and earnestly, but calmly and dispassionately, and show that you are not influenced by those who enforce an argument by sarcasm and ridicule, when reasoning fails to convince, and facts are on the opposite side.

The author of this review in one single sentence contradicts observations based upon careful work, accumulated with anxious care, and confirmed by the statements of several independent witnesses. He sets at nought observations in clinical wards spread over a period of thirty years, and proceeds to deny the truth of the facts upon which a great clinical teacher founded the principles which he taught for nearly a quarter of a century,- principles which have been practically acted upon, and the truth of which has been confirmed in the course of a most active professional career. And if the statements of this reviewer be received, the conclusion is forced upon us, that Dr. Todd was totally deceived in the results of his practice, misled by his observations, mistaken in his views, and prejudiced in favour of a doctrine which was not supported by facts; or we must accept another alternative which is only ungenerously hinted at, because it is well known to be ontradicted by facts, and to be inconsistent with what is known of Dr. Todd's whole career.

I would ask, was it likely that a man who considered him. self to be, and worked till the last at his profession, as a student, was so utterly mistaken in his views, and so completely deceived in the results of his treatment, and wrong in his judg. ment, as this reviewer concludes? Were those house physicians, and clinical clerks and students, who watched during a period of more than twenty years his practice in this Hospital, and the practitioners in this country who act upon principles similar to those on which he based his practice,-are all these, and they must number many hundreds of some of the most inquiring minds in our profession, so utterly mistaken in their impressions, that a quick weak pulse becomes slower amd stronger under the influence of alcohol; and that delirium increases as a patient's strength fails, and diminishes when his strength is upheld? Is it likely that these phenomena, which could be observed again and again by any unprejudiced person who worked for six months in the wards, were in reality merely due, as the reviewer suggests, to the accidents of the malady, and not to the influence of the alcohol? Did the large quantities of alcohol which many of these patients took exert no effect whatever on the pulse?

This gentleman comments on Dr. Todd's conclusions thus : "Te have no hesitation in declaring, as the result of considerable investigations into this subject, that the rate of mortality of Dr. 'Todd's fever cases was much in excess of any other hospital in the United Kingdom?" If this were all, it would not have been necessary to notice the review, as the experience of most readers would prevent them from giving implicit credit to a general statement of this kind, made anonymously. But, armed with the formidable artillery of numbers, he produces facts in support of his assertions which few would be inclined to contradict; and he draws conclusions from the facts which the ordinary reader would not fail to accept. He says that, " about a year before his death, Dr. Todd handed over to him his case-books from the year 1810 to the year 1858 , for the express purpose of analysing the results of his treatment."

It is unfortunate that these books should have fallen into the hands of one so well able to analyse, and so unfortunate in his efforts to extract the truth. After making a most laborious analysis, he makes out that the deaths of typhus, typhoid, and relapsing fever, amounted to 18*29 per cent., while in other hos. pitals the mortality never exceeded 14 per cent. The deaths of Dr. Todd's cases of typhus fever reached 26 per cent. Now, I shall have no difficulty in showing you that all this calculation has been worse than useless, because it has caused the reviewer to propagate a most unpardonable error. His analytical work doubtless is right; but his conclusions are utterly wrong, for the simple reason that the facts upon which his calculations were based are incomplete. He assumes, what he ought to have ascertained before he began his inquiry, that the case-books he analysed contained reports of all the fever cases admitted under Dr. Todd in the above mentioned period. It is hardly credible that so simple a point was not at once determined by questioning those who were familiar with the manner in which the cases were recorded.

[To be concluded next week.]

\section{Bariginal Communitations.}

\section{TEN YEARS OF OPERATIVE SURGERY IN THE PROVINCES.}

By Augustin Prichard, Esq., Surgeon, Clifton, Bristol.

\section{II.-Operations on the Abdomen and Lower Intestine.}

THE second subdivision of my cases includes operations upon the abdomen and the lower part of the alimentary canal; that is, hernia of various kinds, tapping the abdomen, and operations for fistula in ano, hemorrhoids, and fissure of the anus.

Hernia. In narrating the cases of strangulated hernia requiring operation, I shall arrange them according to their kind, namely, femoral, inguinal, and umbilical; describing under each head, first the successful, and then the fatal cases. Of the large majority of cases which were reduced by the taxis, I have no note. A few instances of the operation for the radical cure will be added afterwards.

Femoral Hernia. CASE xLI. F. H., aged about 30, had been the subject of femoral hernia of the right side for two years, the tumour having appeared suddenly one day whilst kneeling at church. Strangulation came on without any very marked effort, but pain and vomiting commenced at once. Calomel and opium were administered, and the patient was bled before I saw her. The loss of blood made her easier, and appeared to lessen the sickness. 'The tumour was as large as an apple. I operated about thirty-six hours after the strangulation occurred; and, finding that I could not pass the fingernail or director under the margin of Poupart's ligament without opening the sac, I punctured it, and let out a considerable quantity of bloody serum. The intestine was very dark, but firm and polished. After dividing the stricture upwards, and exercising a little gentle pressure and manipulation, it was returned. I used two sutures, and a compress and bandage. The patient was relieved of all distressing feeling directly the stricture was removed; and she recovered without a bad symptom of any kind, and was quite well in ten days. There is nothing especially worthy of notice in the case, for everything was plain and straightforward, as if the descriptions in the surgical and anatomical books had been taken from one in every respect similar. It was my first operation for hernia, performed at night, at some distance in the country, away from surgical support; and I was therefore very thankful to meet with no unusual difficulty.

CASE XIII. D. C., aged 59, never wore a truss to relieve a hernial tumour of the right side, from which she had suffered for ten years, and which went back at first, but for the last few years had become permanent. The protrusion increased and became strangulated upon making a violent effort, and severe symptoms began at once. Attempts of various kinds were made to reduce the swelling, but without avail; and when $I$ saw her, about sixty hours after the accident, the tumour was as large as an orange, hard, firm and tense, red, and very painful and œdematous. The pulse was rapid, and vomiting incessant. The case had indications of having undergone quite enough manipulation in the way of taxis, and $I$ therefore operated; but, owing to the cdematous and tense state of the skin, I was unable to draw it together into a fold as usual. 'The sac was opened, and the stricture divided upwards and inwards. The contents were, besides a small quantity of dark bloody fluid, a coil of intestine quite black, but smooth and polished, and a considerable portion of omentum, some of which was hard and thickened, having obviously been in the sac for a great length of time. After returning the intestine, I tied the omentum in strips, and cut off the thick part of it, leaving the ligature hanging out of the wound, which was 
brought together by sutures. She was treated first of all with opiates, and soda water to relieve the vomiting, which still continued. On the second day, some flatus passed from the rectum, showing that the canal was pervious; but she still complained of abdominal pain, and vomited a quantity of green fluid. On the third day, she had an enema of gruel and castor oil, which acted freely, and she was better from this time; being, however, very weak. I gave her six ounces of port wine daily, with the aid of which she soon recovered.

This patient had a very narrow escape; for the œdematous condition of the integuments over the tumour was a sign of mischief within, and the persistence of the vomiting after the reduction of the intestine showed that it had been so pinched that it could scarcely recover itself. The omentum must have acted in a great measure as a protection to the bowel; and, but for its presence in the sac, the efforts at reduction would probably have produced even worse effects than they actually did.

CASE xLIII. J. P., aged 33 , somewhat deformed in the limbs from old rickets, had suffered from hernia of the right side for some years; had never worn a truss; and, four days before her admission, symptoms of strangulation had appeared. She had been constantly sick, and the abdomen was distended and tender. The tumour was of about the size of a walnut, and unusually moveable. After trying the taxis, under chloroform, and finding that it was useless, as it generally is in cases of hernia where the tumour is hard and moveable-for these con. ditions show that it is held by a tight and narrow neck,-I operated, making the first incision by holding the skin over the tumour in a fold, by which means the superficial fascia, as well as the skin, is cleanly divided at first, and the subsequent dissection begins from a known point. After coming down to the sac, I opened it, and some fluid escaped, and what appeared to be intestine was exposed to view. It was smooth and shining and of a pale colour; but it was quite impossible to pass my nail or a probe under the stricture; and, upon drawing firmly the divided edge of the sac, the convex surface of the tumour and the concave surface of the sac were found to be quite continuous with one another ; and it was obvious that another sac was contained within that just opened. This was punctured, and some fluid escaped, and the intestine exposed. It was black, smooth, and polished; and, after some difficulty, I introduced a curved director, and divided the stricture, when the intestine slipped in at once. The after treatment consisted of opiates and rest. The bowels not having been moved by the sixth day, a small dose of castor oil was given; and this had the desired effect. She had retention of urine for three days after the operation, at which time she was menstruating. She was cured in about three weeks.

There are but few cases of double sac recorded, some by Chevallier, and by Dupuytren and Lawrence; and this one which I have just narrated corresponds entirely to those described by these authors. The formation of the double sac is in the following way; the original hernial sac becomes cut off by adhesion from the rest of the peritoneal cavity, and another protrusion takes place exactly at the same spot.

CASE Xliv. S. T., a little fat old woman, aged 66 , had not been subject to hernia, but it came on suddenly two days before her admission, when, besides the tumour in the right groin, she compiained of constipation, frequent vomiting, and general fever. After trying the taxis before and after the ad. ministration of chloroform, I operated, and, opening the sac, divided a very tight stricture; and the intestine, which was dark, slipped back at once.

She had an opiate immediately; but the bowels were open soon after operation. After this, she went on well for some days, still having a little fever, and the inguinal region still being tender; and on the eighth day, the wound looked sloughy, some shreds of tissue hanging out of it. The next day, very fetid discharge, with air and semifluid matter having the appearance and odour of faces, flowed out, and she appeared easier. The bowels, however, were regular, and this unnatural discharge ceased in a few days; and, in three weeks from the operation, the wound was nearly healed. She remained in the Infirmary for two weeks longer, and then was discharged cured.

The large quantity of fat in the subcutaneous cellular tissue rendered this operation a little more difficult than operations for hernia usually are, and the stricture was extremely tight. There was nothing besides the tightness of the stricture to account for the troublesome symptoms that followed, and they might have dependea upon a small slough of the intes. tine, or, what was more probable, upon sloughing of the sac itself, an accident we occasionally meet with.
CASE xLv. M. S., aged 48 , had been accustomed to wear a ? truss ; but one day it happened to be broken, and, whilst lifting $\equiv$ a bucket, the tumour appeared, and she could not reduce it. $S$ I saw her on the third day, and then she was vomiting; her bowels had not been moved; and the abdomen was becoming tender. I gave her a turpentine enema, and applied ice over the tumour; and then tried the taxis, under chloroform, but with- $\frac{\sigma}{7}$ out avail. I therefore operated, and opened the sac, finding $\mathbb{D}$ within a piece of distended colon, with omentum. The tumour was as large as the fist. After dividing the stricture as usual, os so that the finger could pass up into the abdomen, I tried to $\overrightarrow{0}$ return the intestine, but in vain; and it required a second divi- . sion before the hernia could be reduced. I also returned all $\rightarrow$ the omentum, and applied three sutures, compress, and $\omega$ bandage; and ordered her an opiate at once, and again at night. On the third day, the tumour was as large as ever, the omentum having come down again. She slowly mended for a week afterwards, and then went home, because her husband of was very ill.

CASE XlVI. M. W., aged 50, had been the subject of femoral is hernia of the right side for twenty years; and, eight years before I saw her, she had been operated on for strangulation of the intestine, since which time the disease had been irre- $\omega$ ducible. Three days before I saw her, as she was coughing, the swelling increased and became painful, and began to discharge ; and, upon examination, I found a large and irregularly lobulated oval tumour extending across the upper part of the right thigh, and overlapping Poupart's ligament. The abdomen was tense and painful. Upon the outer side of the protrusion was an ulcerated hole, through which serum flowed freely. She had vomited, and the bowels had been confined. D I operated, and opened the sac and integuments to the extent of nearly an inch; and, introducing my finger, found the intes- $\infty$ tine healthy in colour, and the opening into the abdomen free. She became extremely collapsed, and appeared to be sinking; and therefore I did not proceed further with the operation, but brought the parts together, and had her put in bed. The next day, she was much worse, her symptoms evidently being unrelieved. I therefore operated again, and made two separate cuts into the tumour; and found the intestine, of which a very large quantity was in the hernia, agglutinated together by recent peritonitis. This I thoroughly broke down with the finger; and, after bringing the wounds together as well as $I \vec{\circ}$ could, I ordered her some calomel and opium in pills. The first effect of the operation was, that she passed flatus per anum, giving great relief; and, after a good night's rest, she gradually recovered, the tumour still existing upon the upper part of the thigh, and the hernial sac and integuments being so firmly adherent to one another, and so thin from the pressure, that the peristaltic action of the contained intestine could be discerned within. She went out cured, supporting the tumour by a firm bag-truss.

The peculiarities of this case were the size and irreducible state of the hernial protrusion, in the lower part of which strangulation from some inflammatory condition had been set up; and this was relieved by the second operation which I performed upon her.

After this time, the patient came into the Infirmary, suffering o from somewhat similar symptoms, which were relieved by purgatives; and about two years afterwards she died.

CASE xuvir. D. F., aged 45 , had suffered from hernia for many years, but never wore a truss. It had been down twenty-four hours before her admission, and moderate efforts at reduction had been made. The tumour was hard and painful, and the other symptoms acute. I applied ice, gave her chloroform, and used the taxis, but ineffectually; and therefore operated when strangulation had existed only twentysix hours. The operation was straightforward in every respect, but the stricture was excessively tight. After the sac was opened, I had some difficulty in getting the blunt curved director underneath Poupart's ligament; but it was satisfactorily done at last; and, immediately upon the division of the fibres, the intestine slipped back. It was very black, but firm and polished. When she awoke from the chloroform, she expressed herself as relieved and easy. Sutures and compress were used. She went on well at first; but, the abdomen continuing tender, I ordered her a grain of calomel and a quarter of a grain of opium every three hours. On the fourth day after the operation, the bowels were moved, and she appeared better; but upon the seventh day, a sloughy substance, which I supposed to be the sac, presented itself at the wound; and this was fol lowed by some fetid yellow matter, which increased very much on the next day, and was obviously fæcal. This continued, at 
one time copiously, at another sparingly, for eleven days, when $I$ gave her a dose of castor oil, and the bowels were moved naturally. The discharge from the groin continued to be fæca until the twenty-second day after the operation, when it ceased finally; and in three days more the wound was healed.

This case of artificial anus is remarkable on several grounds. It shows that, in acute strangulation, sufficient damage may be done to the intestine to cause a slough in some part of its circumference in twenty-four hours ; and that, under favourable circumferences, this aperture will heal of itself. The patient had great difficulty in holding herself upright for a consider able time, owing to the adhesion formed between the intestine and the parietes. I believe that the hernial protrusion reappeared at the same place some time after she went out.

CASE XLVIr. M. C., aged 60 , was admitted with a strangulated hernia, which had appeared first of all two days before, and upon which, the previous day, some forcible efforts at reduction had been made. She had constipation, vomiting, and pain in the tumour and over the abdomen.

In operating, I tried to divide the stricture without opening the sac, but could not succeed in doing so. In the sac was a quantity of bloody gelatinous serum, and a coil of intestine black, collapsed, and rough and ragged upon its surface. Upon touching the stricture with the edge of the bistoury, it gave way at once, and the intestine went back. Believing that this patient also was suffering from peritonitis, I treated her with some calomel and Dover's powder, giving her at the same time a little wine, for her pulse was rapid and weak. Large quantities of serum flowed from the peritoneal cavity, and she suffered from retention of urine. She continued to take mercury and opium for six days, when the mouth became a little sore; and at the same time the pulse became quiet, and the bowels were moved. She was cured in a few days afterwards. The effect of the mercury in this case was very distinct, and it undoubtedly aided in bringing about the good result that followed. The intestine was so much damaged by the effects at reduction, that I was fearful it would slough; and $I$ have seen many deaths from the intestine not recovering its vitality, when apparently it was not worse than in the present instance.

CASE xuIx. M. R., aged about 50, had been the subject of hernia in the left femoral region for some years. It had become strangulated four days before I operated, at which time she was very prostrate and weak, and constantly weeping and vomiting. In the sac I found omentum and a piece of black polished intestine: the latter was returned easily after the divi sion of the stricture; the former was allowed to remain. She had swallowed a large quantity of purgative medicine before the operation; and when the stricture was relieved and the protruded bowel replaced, a troublesome diarrhœa set in, requiring opium and other astringents. She made a speedy and satisfactory recovery.

CASE L. T. B., aged 60, a farm labourer, fell from a hayrick; and, two days afterwards, he found that he had a swelling in the groin, which was very painful. Vomiting commenced the day after the injury, and he was admitted as my patient on the morning of the fifth day, when I found that he had stran culated femoral hernia of the left side; the tumour being red, and the skin œdematous. I cut down upon it; and, before opening the sac, a : quantity of purulent matter flowed out apparently from the cellular tissue outside the sac. The intestine was dark, and tightly pinched in the opening; but the whole circumference of the bowel was not included underneath the stricture. Upon dividing the edge of Poupart's and Gim. bernat's ligament, the hernia was at once reduced.

This patient required stimulants after the operation, to keep him alive; for he was extremely sunk and prostrate. The bowels were moved on the third day, with the aid of two drachms of castor oil and twenty minims of turpentine.

A week after the operation, the sac sloughed out, and the wound soon healed. He suffered from retention of urine, requiring the catheter, during the progress of his cure.

CASE LI. A. M., aged 52, the subject of hernia previously, found an increase in the tumour and a painful state of the abdomen sixty hours before I saw her. The vomiting was distinctly stercoraceous; and the rupture was small and hard, and in the left femoral region. I opened the sac, in which was some very dark fluid; and found a small coil of black polished intestine, which slipped back into its natural cavity immediately upon the division of the stricture. One suture, compress, and handage, were applied. She recovered without a bad symptom the bowels having been opened with castor oil on the fourth day.
CASE LII. C. S., aged 30, had hernia for six years, and had worn a truss; but, seven hours before I saw her, her foot slipped a few inches off a plank, and the sudden jerk to the body brought down the swelling. It was in the right groin, and of the size of a walnut, very hard and painful; and the general symptoms were urgent. I operated about nine hours after the accident, and returned the intestine without opening the sac; and she appeared relieved at once. No bad symptoms followed; the bowels were relieved by two drachms of castor oil on the third day, and she was soon cured.

The following four cases are instances where the operation did not succeed in restoring the patient.

CASE LIII. E. II., aged 69, with strangulated femoral hernia of the left side. The disease had existed for many years, but had been strangulated only twenty-four hours before I saw her. She had vomiting and tenderness of the tumour and abdomen, and attempts had been made to reduce it. Upon operating, I found in the sac a considerable portion of intestine, rough, dark red, and thickened; and it contained hard facal matter. After dividing the lower part of Poupart's ligament, I tried to reduce the mass, but was unable to do so until there had been a second division made internally, involving Gimbernat's liga ment; and then it passed back readily. She was treated witl opiates. The bowels were moved on the third day, the sickness had ceased, and she appeared to be going on well at first; but, after a day or two, she became weak and sinking, with some hiccup, tympanitis, and intermittent pulse; and she died on the seventh day after the operation.

The post mortem examination showed general peritonitis and effusion of coagulable lymph, producing partial allhesion between the contiguous peritoneal surfaces. Eight inches of the ileum, near the valve, were very dark; and, although none of it was sloughing, one part was much thickened, and in a worse condition than it was at the time of the operation. A single tubercle, with a cicatrix of an old cavity, were found in the top of each lung.

CASE LIV. W. J., aged 56, had suffered from strangulation for about eighty hours before his admission. He was very dispirited and weak, and the tumour had been subjected to great pressure in attempts at reduction. I operated without chloroform. The sac contained a portion of very dark and rough intestine; and, after freeing the stricture, it was easily returned. Three sutures, compress, and bandage, were applied. I ordered him an opiate draught every three hours; notwithstanding which, his bowels were moved twice soon after the operation, and he seemed relieved. I ordered him a littie wine, and in about tiventy-four hours he seemed much better. He then suddenly began to sink, and died early in the morning of the third day.

A portion of small intestine was found, at the post mortem examination, to be very dark and adherent to the surrounding parts. There was no general peritonitis, nor effusion, nor any sign of injury about the ring. The intestine had not recovered itself, but it did not appear sufficiently damaged to cause death.

CASE LV. M. M., aged $\% 0$, had suffered from hernia of the right side for some time, and it had been down and strangulated for ninety-six hours; but no efforts to return it had been made, for it liad not been discovered. Upon operating, I made a very small incision, and found within the sac some dark fluid and a coil of black intestine, with a vein containing air upon its surface, and an abrasion of the peritoneum. The stricture was remarkably tight; but, as soon as it was divided, the bowel was very easily returned. 'Two sutures, compress, and bandage, were applied. This patient, having taken large quantities of purgative medicine, with the view to relieve the constipation that had existed for four or five days, was taken directly after the operation with diarrhœea, and she gradually sank, and died on the sixth day. No post mortem examination was made.

CASE IVI. M. A. F., aged 48 , with strangulated femoral hernia of the right side, of an oblong form, projecting over Poupart's ligament, which had been strangulated for ninety hours. It was discovered to be the cause of her sufferings, but she would not submit to be operated upon before. The symptoms were very urgent, but she was in a weak and collapsed state. I divided the stricture fairly, without opening the sac; but could rot return its contents, and therefore I opened it, and found a mass of omentum, and underneath a portion of rough black intestine, of a tolerably firm consistency. The finger passed up through the neck of the sac, which was not divided; and the intestine passed up readily. I also returned the omentum, which appeared to be thicliened by pressure, but not very hard. She was treated with stimulants and opiates; 
bitt she did not rally at all, and died about twenty-four hours after the operation.

Post Mortem Examination. General intense peritonitis; the membrane being red, and the contiguous parts universally adherent; the lower parts of the abdomen and pelvis were full of serum and flakes of pus. The intestine that had been strangulated had not recovered itself, but it was not sloughy.

$$
\text { [To lie continued.] }
$$

\section{CASES* OF TUBERCULAR MENINGITIS: WITH REMARKS.}

By William Rominson Hiri, M.D.Fuin., one of the Hon. Med. Ofticers to the Eastern Dispensary, Bath; Ext. Mem. and formerly President of the Roy. Med. Soc., Edin.

Tine pathology, diagnosis, and treatment of tubercular meningitis, are points of great interest and importance; and the comparative rarity of the disease, in adults at least, will, I hope, warrant me in bringing the subject before you.

As evidence of this, I may mention that these were the only cases that occurred out of about three hundred phthisical patients that came under my own immediate observation in the course of a vear at the Brompton Hospital for Consumption.

Of these three hundred, about two-thirds were of the male sex, and in each case the subject of the attack of meningitis was a man; therefore, I imagine we may fairly conclude that the disease, though affecting only a very small per centage of consumptive patients, is more common in males than in females. Without further preface, I shall give the details of the more interesting features of the three cases, and conclude with some remarks on certain points which seem to be illus. trated by their history.

C.SSE I. R. P., aged 38, a footman, born in Norfolk, and living in London, but of Irish extraction, was admitted into the Hospital for Consumption at Brompton, on June 3rd, 1858. He was of a consumptive family, and had been subject for some years to a winter cough, leaving him in summer. At the time of his admission, he had courhed only for six or eight weeks, and had been affected also with dyspnœa and considerable pain in the left side, increased by deep inspirations. During the latter half of the period, the cough was accompanied by a little expectorntion, and on one occasion by slight hæmoptysis. His appetite had fallen off and he was losing flesh rapidly. His pulse was small and frequent, 120 per minute, and the respirations 10 per minute. He complained of headache, from which he had suffered more or less for two months.

As regards the state of the lungs, with the exception of some marked duiness and absence of respiration at the left base, indicating the presence of fluid in the pleura, the physical signs were vague. The respiratory sounds were harsh at some points, and accompanied generally throughout both lungs by sibilant rhonchus. Small crepitation was also heard in both lungs at different points.

'Two days after udmission, I noticed that whilst the patient's right pupil was of considerable size, the left one was very much more dilated, but further than this there was no other perceptible change in his condition, either physical or mental. On the day after, the left pupil remaining as fully dilated as formerly, the right one was noticed to be even larger still, so that that iris existed merely as a narrow rim. Neither of the irides showed any contraction on sudden exposure of the eyes to a strong light.

Moreover, there was ptosis of the right upper eyelid with paralysis of the smperior, inferior and internal recti muscles of the right eyelall, indicated by strong and motionless divergence of that eyeball towards the outer canthus. The con dition of his mental faculties varied, as at some periods of the day he was quite rational, at others he was talking nonsense, and sometimes he was insensible. In his rational moments he declared the pain in his head was not so great as formerly. His pulse was still at 120 , but increased in volume. The tongue was dry and heat of skin great. His urine was exam. ineil, and found to be of specific gravity 1025 , of neutral reaction, and speedily becoming alkaline and depositing phosphates on the application of heat; not containing albumen.

He was ordered a mercurial pill to be given three times a day and a blister to be applied behind each ear. The blisters rose

* The first two cases were patients of Dr. Quain, and the third was under Dr. W. Smith, to both of which gentlemen I atn indebted for courteous permission to make use of the notes I took of their history and treatment. well, but the patient's delirium and insensibility continued to increase. Paralysis of the muscles supplied by the third nerve on the left side also supervened, as well as could be made out as the divergence affected both eyes. He had retention of urine also, and some tympanitic distension of the abdomen. $\mathrm{He}$ con:tinued to sink rather rapidly, and died on the fourth day afte the first dilatation of the pupil occurred.

Autopsy. Head. There was an unusual amount of cerebro spinal fluid. The arachnoid on the upper surface of the braic was opaque and gelatinous-looking. At the base it was muct thickened, and contained little masses, which, with the micrf scope, were seen to consist of small granular cells, such as are always seen in the tubercles of the meninges. Each laterd ventricle contained about an ounce of serum. The brain sub. stance appeared normal throughout. The third nerve on the right side was of a dark red colour, for the distance of about one inch, commencing near its origin. It was also thicker and firmer than natural at that part. The left nerve seemed to of normal structure.

Thorax. There were some, not very firm, pleural adhesion on the right side over the diaphragm, also at the apex and along the anterior margin of the left lung. The left pleura contained about a quart of dark red clear serum.

The right lung was infiltrated with small hard miliary tubef cles, though its substance was crepitant and floated well in water. It was also congested. The left lung had much tioe same characters.

The pericardium contained about an ounce of serum. Tho heart weighed nine ounces and was quite healthy.

Abdomen. The liver weighed three pounds eight ouncest and appeared normal. The kidneys and spleen were also healthy, but congested.

CASE II. P. M'L., aged 33, a labourer, of Irish parentage, living at Westminster, was admitted on February the 9th, 1859 $\mathrm{He}$ knew of no hereditary tendency to consumption, but hat been addicted to drink and prize-fighting. The only histort he could give of himself was, that he had had a slight cough and had been losing flesh for two years, though he stated that he had had hæmoptysis to the amount of four quarts seven years previous to admission and to a less extent subsequen on four or five occasions. He had had no other illness. Hs appetite was good, tongue pretty clean, and pulse below $100 . \overline{\bar{O}}$

On physical examination of the chest there was distingt evidence of a cavity at the apex of the right lung with softeniogs extending low down, and a rather harsh character of tipe respiratory sounds with a shade of dulness indicated the pre sence of a small amount of deposit at the left apex.

He was treated with cod-liver oil and counter irritation iodine under the right clavicle, and with the exception of ome slight attack of hæmoptysis progressed favourably during the first fortnight, after which time he began to complain of fecting very ill with much headache, and he soon began to be ve्sy restless at night, constantly getting out of bed, and unableso micturate. On March 2nd, the catheter was used, and this was continued as often as required, to the day of his death. The urine, on examination, was found to be of specific gravity 1011 , of alkaline reaction, and containing albumen in soae quantity. Two days after, there was partial inability to speen, though the patient seemed quite sensible. The pupils were equal and contractile. On the following morning, he was quite unable to speak, and was only partially sensible. There fols slight divergent squint with great dilatation, and immobility of the left pupil, the right one remaining only moderaty dilated. His pulse was 144 per minute, and very weak. \#n the evening of the same day, the left pupil was a little smaher than it had been in the morning, and the right one was tisn larger than the left. 'They were both immobile.

On the next day, he was much weaker and quite insensige. Both pupils were much dilated, the left being a little ffoe largest. There was apparently no other paralysis. He continued to sink, and died early the next morning. The of treatment adopted during the last week of this man's life the frequent administration of small quantities of the mist. $\mathrm{sp}$. vir. gall. together with other measures for supporting $\bar{\Phi}$ is strength.

Autopsy. Head. The dura mater and arachnoid Wre rather unu adherent to one another. About two ounes of cerebro-spinal tluid escaped. The surfaces of the lobesin the fissure of Sylvius were very adherent to one another, and there was much opacity of the surface of the hemispheres ffom subarachnoid effusion. The membranes round the rootso of the nerves at the base of the brain were congested, thicked, and opaque, containing little hard masses, the size of 